\title{
Modeling of Refractive Index Sensing Using Au Aperture Arrays on a Bragg Fiber Facet
}

\author{
Gongli XIAO ${ }^{*}$ and Hongyan $\mathrm{YANG}^{2}$ \\ ${ }^{1}$ Guangxi Key Laboratory of Precision Navigation Technology and Application, Guilin University of Electronic \\ Technology, Guilin 541004, China \\ ${ }^{2}$ School of Electronic Engineering and Automation, Guilin University of Electronic Technology, Guilin 541004, China \\ *Corresponding author: Gongli XIAO E-mail: xgl.hy@163.com
}

\begin{abstract}
A finite-difference-time-domain (FDTD) approach is undertaken to investigate the extraordinary optical transmission (EOT) phenomenon of Au circular aperture arrays deposited on a Bragg fiber facet for refractive index (RI) sensing. Investigation shows that the choice of effective indices and modal loss of the Bragg fiber core modes will affect the sensitivity enhancement by using a mode analysis approach. The critical parameters of Bragg fiber including the middle dielectric RI, as well as its gap between dielectric layers, which affect the EOT and RI sensitivity for the sensor, are discussed and optimized. It is demonstrated that a better sensitivity of $156 \pm 5 \mathrm{~nm}$ per refractive index unit (RIU) and an averaged figure of merit exceeding 3.5 $\mathrm{RIU}^{-1}$ are achieved when $\mathrm{RI}$ is 1.5 and gap is $0.02 \mu \mathrm{m}$ in this structure.
\end{abstract}

Keywords: Optical fiber sensors; surface plasmon resonance; periodic array; refractive index sensing; finite-difference time-domain

Citation: Gongli XIAO and Hongyan YANG, "Modeling of Refractive Index Sensing Using Au Aperture Arrays on a Bragg Fiber Facet," Photonic Sensors, 2019, 9(4): 337-343.

\section{Introduction}

Surface plasmon resonance (SPR) is the resonant oscillation of free electrons excited by light at the metal/dielectric interfaces [1]. Due to the interfacial nature of SPR, refractive index (RI) sensing for quantitative analysis of chemical reactions and biological interactions has become one of the most promising applications of nanoplasmonics. Thus, SPR is adopted in many optical tools for measuring light-matter interactions onto the surface. In the first place, SPR on metamaterial absorbers as sensing has been focused on optimizing the metallic nanoparticle geometries to improve the sensor performance [2-5]. Afterwards, SPR on periodic metallic structures has attracted increasing interest since the observation of extraordinary optical transmission (EOT) phenomenon being first demonstrated by Ebbesen in 1998 [6]. A new generation of the optical sensor based on EOT has been regarded as a promising solution for RI sensing purposes. Like that, exploring the SPR electromagnetic field localization properties for sensing has been proposed to overcome the limitations in operation and beat performances of other optical detection approaches [7]. In contrast to prism-based SPR sensors with Kretschmann configuration [8], the combination of SPR and optical fiber would have the possibility for far extending the scope of SPR utilization in biological

Received: 10 October 2018 / Revised: 11 January 2019

(C) The Author(s) 2019. This article is published with open access at Springerlink.com

DOI: $10.1007 / \mathrm{s} 13320-019-0542-0$

Article type: Regular 
and chemical communities. Over the past few years, many fiber-based SPR sensors have been reported, including SPR sensor configurations with multimode, single mode, and D-shaped fibers coated with a thin metallic layer [9-11]. These kinds of sensors require a complex manufacturing process, and their sensing performances are not good. Owing to the impressive progress in the nanofabrication technology, many researchers have pursued this idea in a quest to create sensitive RI sensors by fabricating an apertures array integrated on optical fiber facet. More recently, there have been many successful attempts to realize SPR based EOT sensors in optical fibers facet [12-16]. However, these early proposals offer preliminary designs with little theoretical or experimental evidence to show that the sensing performance of optical fiber sensors would be enhanced by a metallic nanostructure. We would like to develop some new types of nanostructures that can improve the analytical figures of merit (FOM), such as detection limits and sensitivity, relative to the commercial systems.

In this paper, we report a novel device, which is composed of $\mathrm{Au}$ aperture arrays directly fabricated on the core-cladding Bragg (C-C Bragg) fiber facet and it is applied as an optical sensor based on EOT. We begin with the comparative analysis of effective indices $\left(n_{\text {eff }}\right)$ and loss of the core modes for C-C Bragg fiber and $\mathrm{C}-\mathrm{C}$ fiber by using the mode analysis approach. Here, the effect of crucial parameters of C-C Bragg fiber including the middle dielectric RI $\left[n_{(M)}\right]$, as well as its gap $\left(t_{g}\right)$ between dielectric layers on the EOT, is analyzed and optimized numerically using the finite-difference-time-domain (FDTD) method. Moreover, by tuning the above two parameters, we can obtain the best EOT property and increase its sensitivity. We hope our findings provide guidance to fundamental research of a high-sensitivity integrated optical fiber sensor based on EOT.

\section{Device structure and the analysis method}

The schematic diagram of the proposed optical sensor with $\mathrm{Au}$ circular aperture arrays on a C-C Bragg fiber facet (RI of core is denoted by $n_{1}$; RI of cladding is denoted by $n_{2}$ ) is shown in Fig. 1(a). Au aperture arrays are placed onto the fiber facet, where the pitch of the square lattice is denoted by $\Lambda$, $\Lambda=0.4 \mu \mathrm{m}$ and the diameter of air aperture is denoted by $d_{a}, d_{a}=0.2 \mu \mathrm{m}$. The Bragg fiber, where the high index central region (acts as the core) is surrounded by concentric layers of alternate low refractive index materials, is shown in the top view structure of Fig. 1(b). Figure 1(b) shows the refractive index variation with respect to the radial distance for the rectangular solid core Bragg fiber, in which $d_{c}$ is the diameter of the solid core region, and $n_{(M)}$ and $t_{g}$ are the RI of two different consecutive cladding and its gap, respectively.

Afterwards, we use the commercial full-wave electromagnetic field simulation software package, FDTD Solutions and MODE Solutions (Lumerical Solutions Inc., Canada) [17] to investigate the modal profiles, EOT, and sensing performances of the proposed sensor, which uses finite difference approximation in both time and space domains to calculate the Maxwell's curl equations step by step. Initially, $\mathrm{Au}$ aperture arrays are filled with air, $n_{\text {air }}=1.0$. RIs of the C-C fiber are taken as $n_{1}=2$ and $n_{2}=1.5$, respectively. The dielectric constant of $\mathrm{Au}$ in the visible and near-IR region is defined by the Drude model described as [18]

$$
\varepsilon_{\mathrm{Au}}(\omega)=\varepsilon_{\infty}-\omega_{p}^{2} / \omega\left(\omega+\mathrm{i} \omega_{c}\right)
$$

where $\varepsilon_{\infty}=9.75$ is the dielectric constant of Au at high frequency, $\omega_{p}=1.36 \times 10^{16}$ is the plasma frequency of $\mathrm{Au}, \omega_{c}=1.45 \times 10^{14}$ is the scattering frequency of electron, and the data were given by Johnson and Christy [19]. In our calculation, we use mesh sizes ranging from $1 \mathrm{~nm}$ to $4 \mathrm{~nm}$. For the calculation of the transmittance, the structures are excited by a mode sources packet composed of normally incident single fundamental mode waves (with the electric field pointing along one of the axes of the square array) and all frequencies of interest in a small solid angle centered around the normal 
direction along the $z$-direction. Infinite periodic aperture arrays are simulated by applying Bloch conditions at the boundaries of the unit cell and imposing "uniaxial perfectly matched layer (PML)"
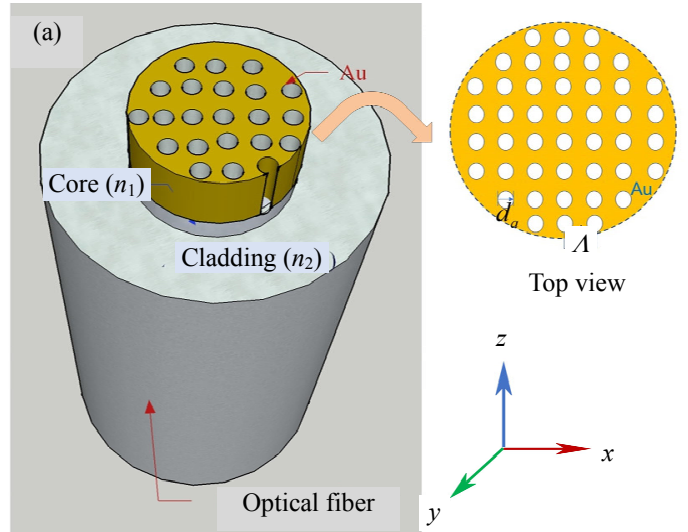

at surfaces parallel to the Au film. The calculated zero-order transmission spectra around the circular apertures are shown in Figs. 3(a), 3(b), Figs. 4(a), 4(c), and Fig. 5(a).

(b)

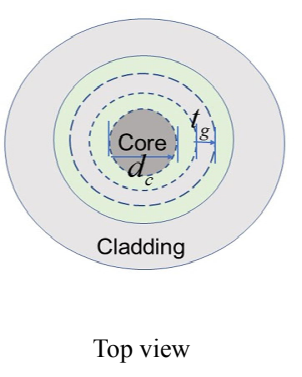

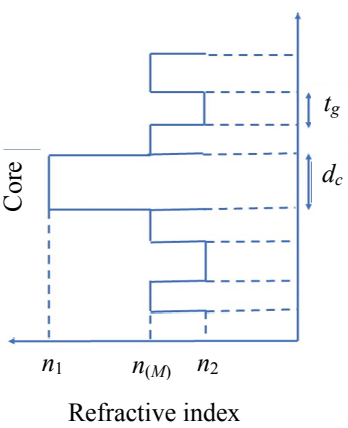

Fig. 1 Schematic diagram of the optical sensor with Au circular aperture arrays on a C-C Bragg fiber facet: (a) 3D view of the structure and (b) top view of the structure and RI variation with radial distance of rectangular solid core Bragg fiber.

\section{Results and discussion}

In order to gain a deeper understanding, we analyze the dependence of the optical sensor mode's $n_{\text {eff }}$ for C-C Bragg fiber and C-C fiber using a finite-difference eigenmode (FDE) solver of MODE Solutions. Figure 2(a) depicts the variation tendency of their real parts of $n_{\text {eff }}$ as a function of the wavelength. The real part of $n_{\text {eff }}$ decreases with increasing wavelength corresponding to C-C Bragg fiber and $\mathrm{C}-\mathrm{C}$ fiber. And it is found that the latter decays faster. The illustration shows their corresponding index profile in $x-y$ plane. By studying comparatively, we suggest that C-C Bragg fiber is the optimal structure. To test the idea, we also analyze the dependence of the optical sensor mode's modal loss for C-C Bragg fiber and C-C fiber. The fundamental mode is analyzed to investigate the propagation loss. By using the imaginary part of effective index $\operatorname{Im}\left(n_{\text {eff }}\right)$, the propagation loss is defined as [20]

$$
\begin{aligned}
\alpha & =40 \pi \operatorname{Im}\left(n_{\text {eff }}\right) /[\ln (10) \lambda] \\
& \approx 8.686 k_{0} \operatorname{Im}\left[n_{\text {eff }}\right] 10^{4} \mathrm{~dB} / \mathrm{cm}
\end{aligned}
$$

where $k_{0}=2 \pi / \lambda$ is the wave number in the free space, and the wavelength and $\lambda$ is in micron.
Figure 2(b) shows the dependence of the modal loss spectra of the fundamental mode for C-C Bragg fiber and $\mathrm{C}-\mathrm{C}$ fiber. It is found that the leaky energy loss exhibits an upward positive enhancement trend for C-C Bragg fiber and a downward negative enhancement trend for $\mathrm{C}-\mathrm{C}$ fiber, which indicates the effective occurrence of SPR in C-C Bragg fiber.

To investigate the RI sensing property of the proposed sensor, we firstly use an FDTD numerical simulation to model the EOT spectra of Au aperture arrays on C-C Bragg fiber and C-C fiber two facets. Structure parameters include $n_{1}=2, n_{2}=1.5$, $n_{(M)}=1.8$, and $t_{g}=0.03 \mu \mathrm{m}$. Then, their RI sensing properties are analyzed. The simulated results are presented in Fig. 3. Figure 3(a) clearly shows the obvious difference of zero-order transmittance spectra in the wavelength range of $0.4 \mu \mathrm{m}$ and $0.65 \mu \mathrm{m}$ with two different structures. In the first place, it is found that their peak intensity $\left(T_{\text {peak }}\right)$ and full-width-at-half-maximum (FWHM) values are different. Comparing two different $\lambda_{\text {peak }}, 551 \mathrm{~nm}$ and $560 \mathrm{~nm}$, it is found that a higher and narrower solid-line peak is obtained with C-C Bragg fiber. It shows that the solid-line $T_{\text {peak }}$ value is up to 5 arbitrary units $(\sim 40 \%)$ more than that of dashed-line. The solid-line FWHM value is $31 \mathrm{~nm}$, 
less than $47 \mathrm{~nm}$ of dashed-line FWHM value. Meanwhile, it can be found that for $\lambda_{\text {peak }}$ of $551 \mathrm{~nm}$, the $|E|$ distributions in $x-y$ plane are much stronger than that at the $\lambda_{\text {peak }}$ of $560 \mathrm{~nm}$, with a balanced view, C-C Bragg fiber structure shows a better EOT performance. Then, we perform an RI sensing analysis of the optical sensor with C-C Bragg fiber and $\mathrm{C}-\mathrm{C}$ fiber by calculating their zero-order transmission spectra in $n_{s}=1.30$ and $n_{s}=1.38$, as shown in Fig.3(b). The inset of Fig. 3(b) shows the $|E|$ distributions of fundamental core mode and SPP mode in $x-z$ plane at their corresponding $\lambda_{\text {peak }}$. With a C-C Bragg fiber added, the fundamental core mode and SPP mode strongly couple together, the $T_{\text {neak }}$ observably enhances, meanwhile, $\lambda_{\text {neak }}$ has a

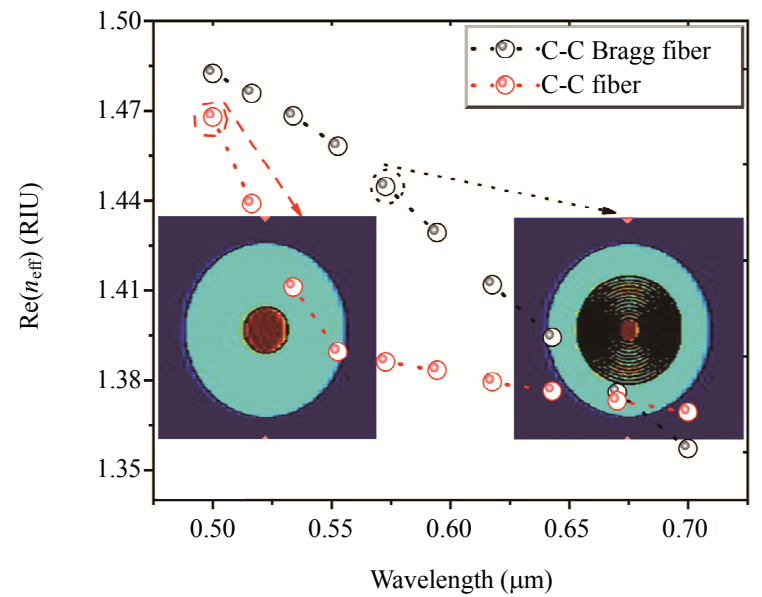

(a)

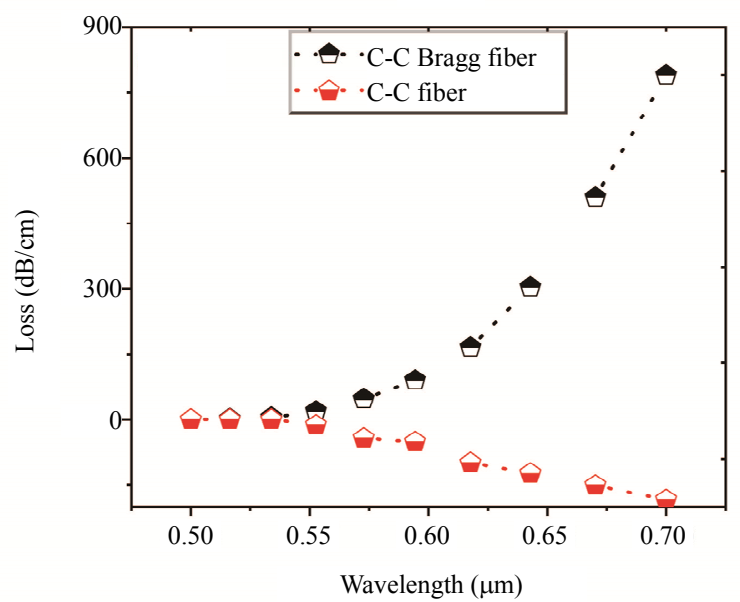

(b)

Fig. 2 Model analysis for (a) the real parts of $n_{\text {eff }}$ and (b) modal loss of fundamental mode with C-C Bragg fiber and C-C fiber as a function of the wavelength, calculated for $n_{1}=2$ and $n_{2}=1.5$. large range redshift ( $\left.\Delta_{1}=18 \mathrm{~nm}>\Delta_{2}=8 \mathrm{~nm}\right)$, which leads to the improved sensitivities in wavelength interrogations. The proposed optical sensor operation is based on the SPR evanescent fields and its interaction with the analytes. In view of the experiment, to determine the sensitivity, gold hole array fiber is equipped with a fluidic channel. The analyte is injected into gold hole array through the flow cell sequentially using a syringe pump. The sensitivity is analyzed by using the $\lambda_{\text {peak }}$ interrogation method. The corresponding sensitivities are expressed in [20], which are given in units of $\mathrm{nm} / \mathrm{RIU}$

$$
S_{(\lambda)}=\Delta \lambda_{\text {peak }} / \Delta n_{s}(\mathrm{~nm} / \mathrm{RIU})
$$

where $\Delta \lambda_{\text {peak }}$ is the resonance peak shift, and $\Delta n_{s}$ is the variation of the RI of analyte.

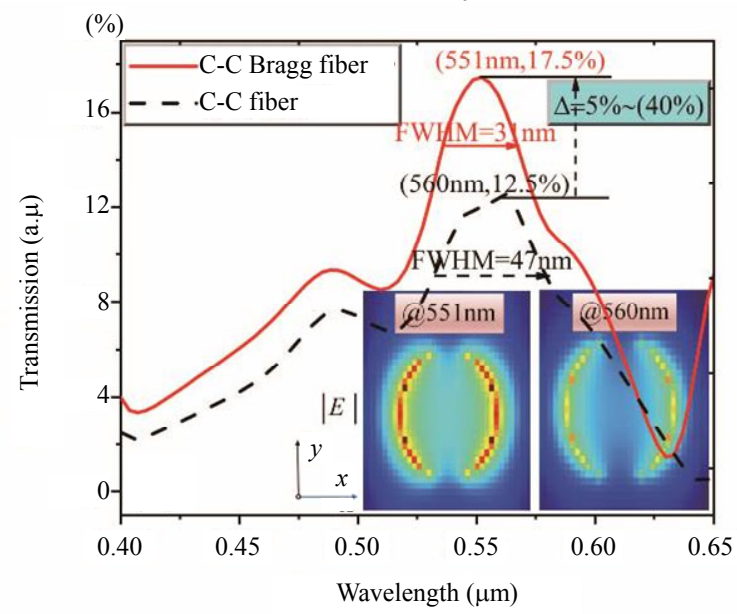

(a)

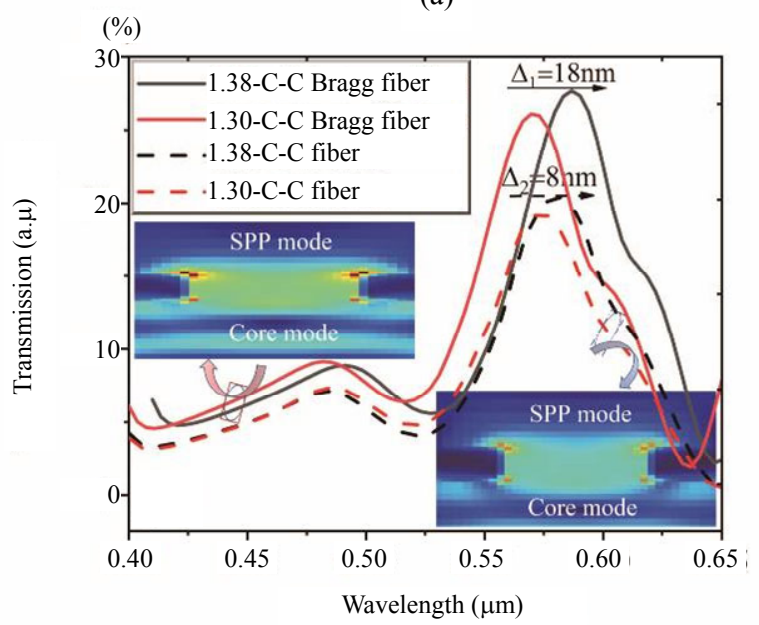

(b)

Fig. 3 Calculated zero-order transmission spectral as a function of the wavelength: (a) with C-C Bragg fiber and C-C fiber and (b) for their two different analyte RIs (1.30 and 1.38). 


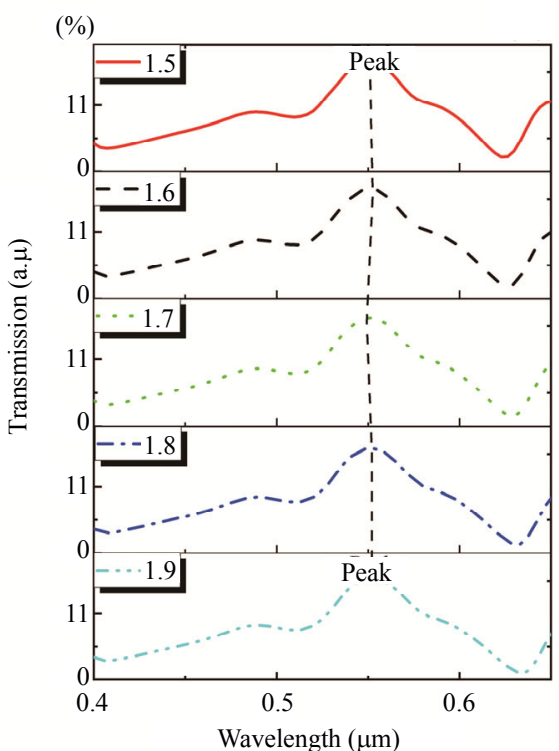

(a)

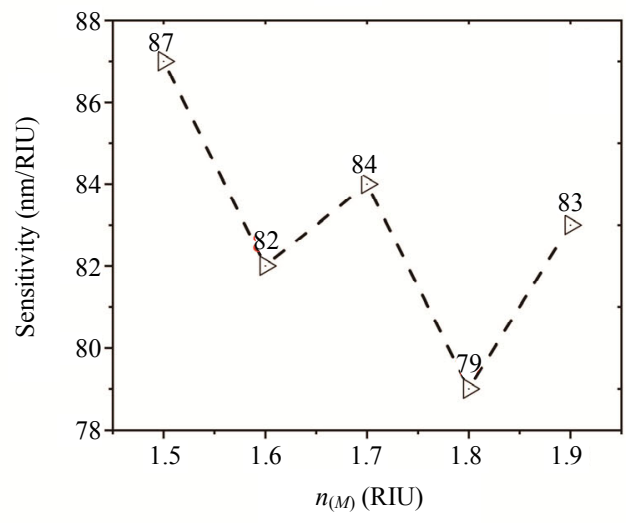

(c)

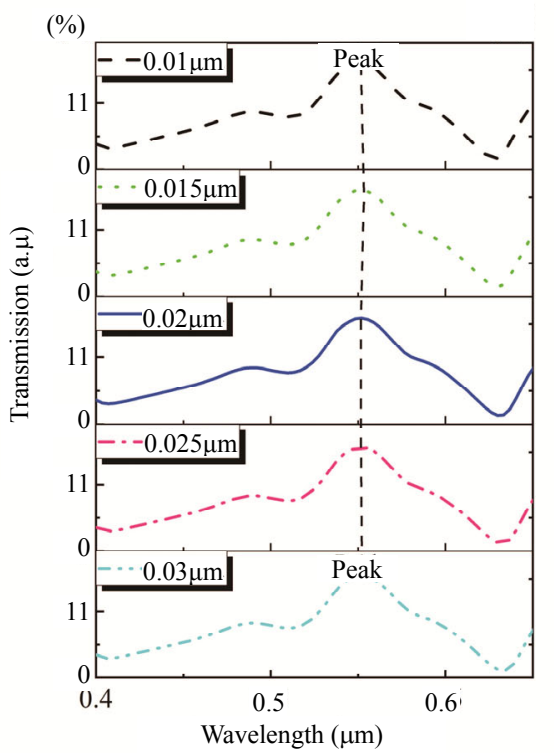

(b)

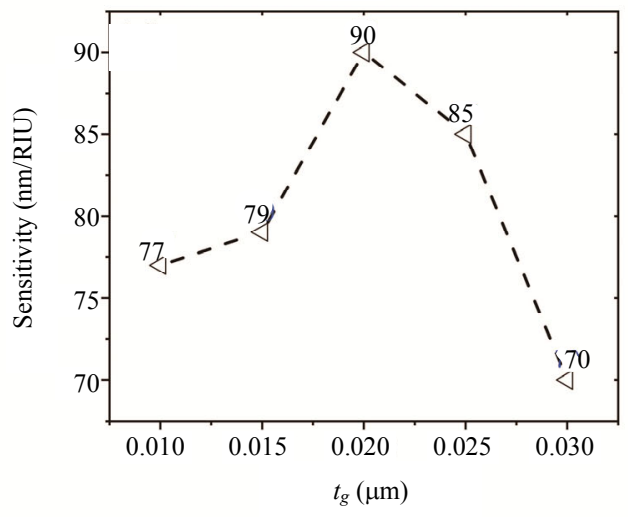

(d)

Fig. 4 Calculated zero-order transmission spectral dependence on different (a) $n_{(M)}$ and (c) $t_{g}$ as a function of the wavelength, the dashed black arrows indicate the shift of the different $\lambda_{\text {peak }}$; sensitivity versus (b) $n_{(M)}$ and (d) $t_{g}$.

Firstly, $n_{(M)}$ is introduced to optimize the EOT performance. Figure 4(a) plots the calculated zero-order transmission spectra of the sensor with $n_{(M)}$ of $1.5,1.6,1.7,1.8$, and 1.9 , respectively, when the other parameters remain unchanged. Then, we analyze its sensing performance by calculating the zero-order resonant spectra in a large dynamic $1.30 \leq n_{s} \leq 1.38$ range to find the corresponding $\lambda_{\text {peak }}$ (the five-middle peaks connected by a dashed-line). In the following study, we vary $n_{(M)}$ to investigate its influence on $S_{(\lambda)}$, as shown in Fig. 4(b). The maximal value is 87 with $n_{(M)}=1.5$, as shown in the red-solid-line transmission spectral of Fig. 4(a), where it has a best EOT peak performance. Figure 4(c) plots the calculated zero-order transmission spectra of the sensor with the $t_{g}$ of $0.01 \mu \mathrm{m}, 0.015 \mu \mathrm{m}, 0.02 \mu \mathrm{m}, 0.025 \mu \mathrm{m}$, and $0.03 \mu \mathrm{m}$, respectively, when the other parameters remain unchanged. Then, we analyze its sensing performance by calculating the zero-order resonant spectra in a large dynamic $1.30 \leq n_{s} \leq 1.38$ range to find the corresponding $\lambda_{\text {peak }}$ (the five-middle peaks connected by a dashed-line). In the following study, we vary $t_{g}$ to investigate its influence on $S_{(\lambda)}$, as shown in Fig. 4(d). The maximal value is 90 with $t_{g}=0.02 \mu \mathrm{m}$, as shown in 
the blue-solid-line transmission spectra of Fig. 4(c), where it has the best EOT peak performance.

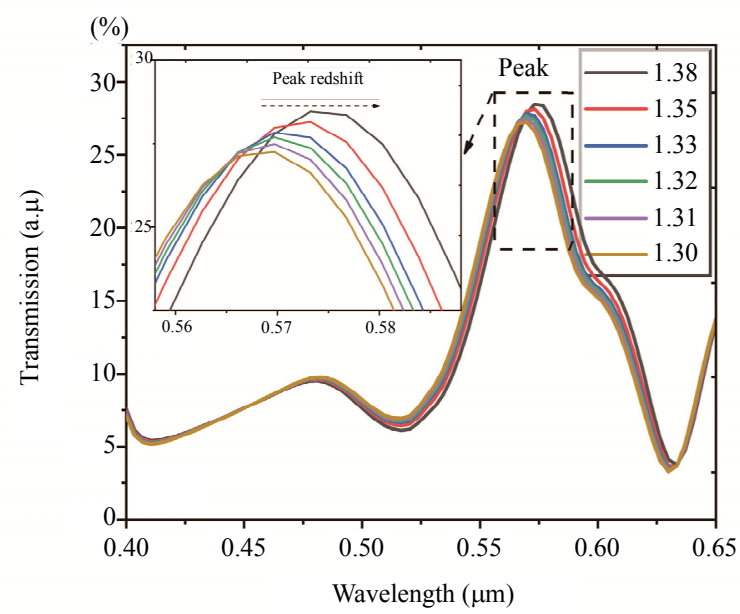

(a)

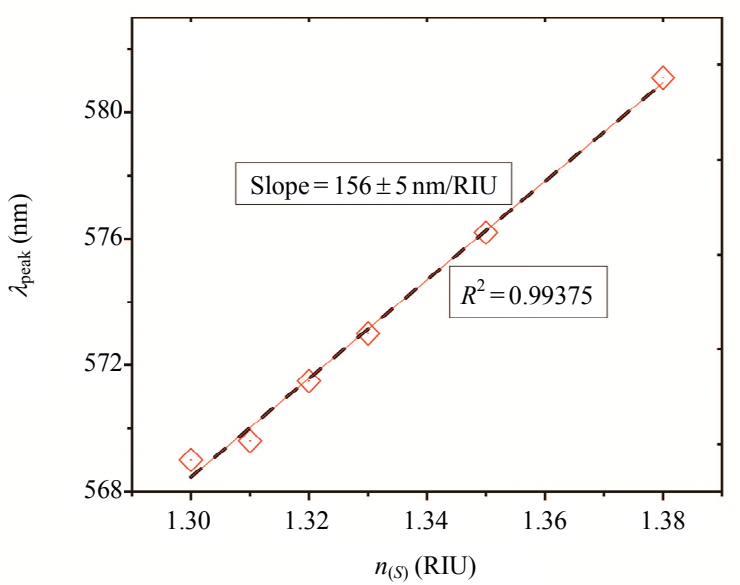

(b)

Fig. 5 Sensor performance analysis for (a) calculated zero-order transmission spectral for the sensor in various $n_{s}$ with $t_{(\mathrm{Au})}=100 \mathrm{~nm}, n_{(M)}=1.5$, and $t_{g}=0.02 \mu \mathrm{m}$, as a function of the wavelength and (b) dependence of the corresponding $\lambda_{\text {peak }}$ on $n_{s}$, showing the linear fitted result of $\lambda_{\text {peak }}$ with different $n_{s}$.

Above findings advise that through a suitable choice of $t_{(\mathrm{Au})}=100 \mathrm{~nm}, n_{(M)}=1.5$, and $t_{g}=0.02 \mu \mathrm{m}$, a feasible narrow peak can be obtained for use as a sensing device for tracking the $\lambda_{\text {peak }}$ with the variation of $n_{s}$. Thus, the proposed sensor with the above one set of parameters is selected to explore its RI sensing capability. Then, we analyze the RI sensing performance by calculating the resonant spectra in a large dynamic $1.30 \leq n_{s} \leq 1.38$ range to find the corresponding $\lambda_{\text {peak }}$. The $\lambda_{\text {peak }}$ undergoes a regular red-shift as the augment of $n_{s}$, as shown in the enlarged illustration of Fig. 5(a). Finally, we investigate the RI sensitivity by analyzing the above $\lambda_{\text {peak }}$. The corresponding linear fitting lines of $\lambda_{\text {peak }}$ with respect to $n_{s}$ is presented in Fig. 5(b). The regression equations in Fig. 5(b) are as follows:

$$
\lambda_{\text {peak }}(\mathrm{nm})=156 n+365,1.30 \leq n_{s} \leq 1.38 \text {. }
$$

The slopes of the equation give an average $S_{(\lambda)}$ of the analytes, i.e., $156 \pm 5 \mathrm{~nm} / \mathrm{RIU}$, within the relevant sensing range. The adjusted R-Square values of $\lambda_{\text {peak }}$ fitting lines are 0.99375 , indicating a high linearity of the optical sensor. For the practical applications, the FOM is another important parameter of the POS to evaluate its sensing performance, which is defined as $\mathrm{FOM}=S_{(\lambda)} / \mathrm{FWHM}$ [21]. In this regard, the averaged FOM values of the POS can reach 3.5 RIU $^{-1}$

\section{Conclusions}

In summary, a study is undertaken to investigate $\mathrm{Au}$ aperture arrays deposited on the $\mathrm{C}-\mathrm{C}$ Bragg fiber facet for RI sensing using the FDTD method. It is demonstrated that the suitable choice of $n_{\text {eff }}$ and modal loss of the core modes for C-C Bragg fiber can increase the sensitivity with mode analysis approach. The effects of $n_{(M)}$ and $t_{g}$ on EOT phenomenon and $S_{(\lambda)}$ are discussed and optimized, correspondingly. It is found that when $n_{(M)}=1.5$ and $t_{g}=0.02 \mu \mathrm{m}$ in this structure, they yield the best EOT phenomenon, in which $S_{(\lambda)}$ is $156 \pm 5 \mathrm{~nm} / \mathrm{RIU}$, and its averaged FOM value is $3.5 \mathrm{RIU}^{-1}$. This investigation will assist in designing structures that maximize the $S_{(\lambda)}$ of a RI sensing optical device.

\section{Acknowledgment}

This work is partially supported by the National Natural Science Foundation of China (Grant Nos. 61465004 and 61765004), the Guangxi Natural Science Foundation (Grant Nos. 2017GXNSFAA198164 and 
2016GXNSFAA380006), and the Guangxi Key Laboratory of Precision Navigation Technology and Application, Guilin University of Electronic Technology Foundation (Grant No. DH201804).

Open Access This article is distributed under the terms of the Creative Commons Attribution 4.0 International License (http://creativecommons.org/licenses/by/4.0/), which permits unrestricted use, distribution, and reproduction in any medium, provided you give appropriate credit to the original author(s) and the source, provide a link to the Creative Commons license, and indicate if changes were made.

\section{References}

[1] R. Karlsson, "SPR for molecular interaction analysis: a review of emerging application areas," Journal of Molecular Recognition, 2004, 17(3): 151-161.

[2] J. Chen, Q. Zhang, C. Peng, C. J. Tang, X. Y. Shen, L. C. Deng, et al., "Optical cavity-enhanced localized surface plasmon resonance for high-quality sensing," IEEE Photonics Technology Letters, 2018, 30(8): 728-731.

[3] J. Chen, W. F. Fan, T. Zhang, C. J. Tang, X. Y. Chen, J. $\mathrm{J}$. Wu, et al., "Engineering the magnetic plasmon resonances of metamaterials for high-quality sensing," Optics Express, 2017, 25(4): 3675-3681.

[4] J. Chen, J. Yuan, Q. Zhang, H. M. Ge, C. J. Tang, Y. Liu, et al., "Dielectric waveguide-enhanced localized surface plasmon resonance refractive index sensing," Optical Materials Express, 2018, 8(2): 342-347.

[5] J. Chen, H. Nie, C. Peng, S. B. Qi, C. J. Tang, Y. Zhang, et al., "Enhancing the magnetic plasmon resonance of three-dimensional optical metamaterials via strong coupling for high-sensitivity sensing," Journal of Lightwave Technology, 2018, 36(16): 3481-3485.

[6] T. W. Ebbesen, H. Lezec, H. F. Ghaemi, T. Thio, and P. A. Wolff, "Extraordinary optical transmission through sub-wavelength hole arrays," Nature, 1998, 391(6668): 667-669.

[7] R. Gordon, D. Sinton, L. K. Kavanagh, and A. G. Brolo, "A new generation of sensors based on extraordinary optical transmission," Accounts of Chemical Research, 2008, 41(8): 1049-1057.

[8] H. C. Zhou, X. Chen, P. Hou, and C. F. Li, "Giant bistable lateral shift owing to surface-plasmon excitation in Kretschmann configuration with a Kerr nonlinear dielectric," Optics Letters, 2008, 33(11): 1249-1251.
[9] J. C. Hsu, S. W. Jeng, and Y. S. Sun, "Simulation and experiments for optimizing the sensitivity of curved D-type optical fiber sensor with a wide dynamic range," Optics Communications, 2015, 341: 210-217.

[10] B. B. Shuai, L. Xia, Y. T. Zhang, and D. M. Liu, "A multi-core holey fiber based plasmonic sensor with large detection range and high linearity," Optics Express, 2012, 20 (6): 5974-5986.

[11] P. P. Jia and J. Yang, "Integration of large-area metallic nanohole arrays with multimode optical fibers for surface plasmon resonance sensing," Applied Physics Letters, 2013, 102(24): 243107-1-243107-3.

[12] P. P. Jia and Y. Jun, "A plasmonic optical fiber patterned by template transfer as a high-performance flexible nanoprobe for real-time biosensing," Nanoscale, 2014, 6(15): 8836-8843.

[13] P. P. Jia, Z. L. Yang, J. Yang, and E. H. Heike, "Quasiperiodic nanohole arrays on optical fibers as plasmonic sensors: fabrication and sensitivity determination," ACS Sensors, 2016, 1(8): 1078-1083.

[14] P. P. Jia, H. Jiang, J. Sabarinathan, and J. Yang, "Plasmonic nanohole array sensors fabricated by template transfer with improved optical performance," Nanotechnology, 2013, 24(19): 195501.

[15] P. P. Jia and J. Yang, "Universal sensitivity of propagating surface plasmon resonance in nanostructure arrays," Optics Express, 2015, 23(14): 18658-18664.

[16] E. M. Zhao, P. P. Jia, E. H. Heike, and H. Y. Li, "Localized surface plasmon resonance sensing structure based on gold nanohole array on beveled fiber edge," Nanotechnology, 2017, 28(43): 435504.

[17] Lumerical Solutions Inc., FDTD Solutions User Manual, Vancouver, BC, Canada, 2011.

[18] C. Liu, L. Yang, X. L. Lu, and Q. Liu, "Mid-infrared surface plasmon resonance sensor based on photonic crystal fibers," Optics Express, 2017, 25(13): $14227-14237$.

[19] P. B. Johnson and R. W. Christy, "Optical constants of the noble metals," Physical Review B, 1972, 6(12): 4370-4379.

[20] A. A. Rifat, G. A. Mahdiraji, Y. M. Sua, R. Ahmed, Y. G. Shee, and F. R. M. Adikan, "Highly sensitive multi-core flat fiber surface plasmon resonance refractive index sensor," Optics Express, 2016, 24(3): 2485-2495.

[21] M. H. Elshorbagy, C. Alexander, and A. Javier, "High-sensitivity integrated devices based on surface plasmon resonance for sensing applications," Photonics Research, 2017, 5(6): 654-661. 